\title{
Impact of Sleep Duration and Quality on the Burden of Diabetes Among Urban and Rural Community Dwellers in Cameroon
}

\author{
Daniel Lemogoum ${ }^{1,2,3, \text { *, William Ngatchou }}{ }^{1}$, Philippe Van de Borne ${ }^{3}$, Basile Essola ${ }^{1}$, \\ Thierry Messomo $^{2}$, Marc Dandji ${ }^{4}$, Jean Paul Degaute ${ }^{3}$, Marc Leeman ${ }^{3}$, Michel P. Hermans ${ }^{5}$ \\ ${ }^{1}$ Faculty of Medicine and Pharmaceutical Sciences, University of Douala, Douala, Cameroon \\ ${ }^{2}$ Cameroon Heart Institute, Cameroon Heart Foundation, Douala, Cameroon \\ ${ }^{3}$ Department of Cardiology, Erasme University Hospital, Université Libre de Bruxelles, Brussels, Belgium \\ ${ }^{4}$ Laboratory of Medicinal Plants Biochemistry, Food Science and Nutrition, Department of Biochemistry, Faculty of Science, University of \\ Dschang, Dschang, Cameroon \\ ${ }^{5}$ Endocrinologyand Nutrition Unit, Cliniques Universitaires St-Luc, UniversitéCatholique de Louvain, Avenue Hippocrate, Brussels, Belgium
}

\section{Email address:}

dlems2002@yahoo.fr (D. Lemogoum)

*Corresponding author

\section{To cite this article:}

Daniel Lemogoum, William Ngatchou, Philippe Van de Borne, Basile Essola, Thierry Messomo, Marc Dandji, Jean Paul Degaute, Marc Leeman, Michel P. Hermans. Impact of Sleep Duration and Quality on the Burden of Diabetes Among Urban and Rural Community Dwellers in Cameroon. Cardiology and Cardiovascular Research. Vol. 5, No. 2, 2021, pp. 88-93, pp. 94-99. doi: 10.11648/j.ccr.20210502.16

Received: May 2, 2021; Accepted: June 3, 2021; Published: June 15, 2021

\begin{abstract}
Background: Sleep disorders are known to be linked with numerous cardiovascular comorbidities including type 2 diabetes mellitus. The prevalence and impact of sleep quality and duration on diabetes in the Cameroonian population is not well established. This study evaluates the isolated and combined contribution of two aspects of sleep (duration and quality) on glucose homeostasis in an urban and rural Cameroonian population. Methods: This was a cross-sectional prospective survey conducted among 249 rural and 250 urban community dwellers in Cameroon aged $\geq 18$ years. Self-reported sleep duration (SD) and quality were evaluated using the Pittsburgh Sleep Quality Index (PSQI). Poor sleep quality was considered for PSQI score $>5$ and short SD was considered $\leq 6 \mathrm{~h}$. Diabetes was considered for fasting blood glucose $>126 \mathrm{mg} / \mathrm{d}$ Land/or use of glucose-lowering medications. Results: Mean age was $36 \pm 12$ years, and men accounted for $39.1 \%$. Frequency of poor sleep quality was $50.3 \%$ and was similar in urban and rural groups $(48.2 \%$ vs $52.4 \%$ respectively, $\mathrm{p}=0.395)$. Short SD was present in $30.5 \%$ of subjects and was more frequent among urban dwellers $(36.1 \%$ vs $24.8 \%$ in rural, $\mathrm{p}=0.006$, respectively). Short SD was significantly associated with diabetes (OR 2.62, 95\%CI 1.38-5.00). Although the frequency of diabetes was higher in participants with poor sleep quality compared to those with PSQI $\leq 5$ (10\% vs $6.5 \%$, respectively), the observed difference was not significant $(\mathrm{p}>0.05)$. The combination of poor sleep quality and short SD was strongly associated with diabetes (OR 2.67 , 95\%CI 1.23-5.79). Conclusion: This survey demonstrates a significant association between short sleep duration as well as the combination of short sleep duration and poor sleep quality with type 2 diabetes prevalence. It is appropriate to consider sleep quality and duration as potentially modifiable variables associated with the presence or management of diabetes in these Cameroonian populations.
\end{abstract}

Keywords: Sleep Quality, Sleep Duration, Type 2 Diabetes, Urban, Rural

\section{Introduction}

Insufficient sleep duration and chronic sleep disturbancesare clearly identified as a major public health problem in developing countries. Both are endemic in adults and children [1-3] and have important consequences in affected subjects, adversely impacting on health, work, and quality of life $[4,5]$. Recent population studies show that 
sleep deprivation and other sleep disorders affect many more people worldwide than previously thought. Data from US populations show an average of $6.8 \mathrm{~h}$ sleep duration per night, which represents a drop of $1.5 \mathrm{~h} /$ night over a century [6]. About $20-30 \%$ of the general populations are thought to suffer from one or more types of sleep disorders, and approximately 50-70 million Americans chronically present with sleep disorders $[7,8,9]$. Among factors involved in this increasing burden of sleep disorders are rapid urbanization, increased prevalence of shift work, longer working days, prolonged commuting times, increased screen watching, and other leisure time activities [10]. All these factors are also at play in low-and middle-incomes countries, including subSaharan Africa.

Many previous studies have linked habitual short sleep duration to major adverse cardio metabolic outcomes, including weight gain, hypertension, obesity, type 2 diabetes mellitus (T2DM), insulin resistance, metabolic syndrome, as well as other cardiovascular (CV) diseases [11-13]. As regards T2DM, a recent meta-analysis showed a $30 \%$ increase in incident diabetes risk in subjects with short sleep duration $[14,15]$. It was also demonstrated in healthy adults that sleep restriction decreased insulin sensitivity [16], thereby inducing insulin resistance [17]. Poor sleep quality and insomnia were also linked to T2DM and insulin resistance [18].

Although there is a wealth of evidence linking short sleep duration with T2DM, most studies did not examine the separate contribution of different sleep aspects in the development of diabetes. Considering the fact that various sleep disturbances often occur together, it is important to distinguish the respective contribution of different sleep aspects as well as their association, especially sleep duration and sleep quality. We therefore aimed to evaluate the isolated and combined contribution of two major aspects of sleep (i.e. sleep duration and sleep quality) on glucose homeostasis and prevalent T2DM in urban and rural Cameroonian populations.

\section{Patients and Methods}

\subsection{Study Design and Population}

This was a cross-sectional prospective survey conducted from November 2014 to May 2015 in a rural and urban population of the littoral region of Cameroon. Urban participants were recruited in Douala city and rural participants were recruited in the rural community of Njombe. Participants of both genders aged 18 years or older were randomly selected from these two communities. Subjects with the following characteristics were excluded: previous diagnosis of obstructive sleep apnea syndrome or restless legs syndrome and being unable to cooperate with physical examination or interview due to mental disorder or physical disability. In all, 499 participants were included (249 participants from the rural area and 250 participants from the urban area).

\subsection{Data Collection and Parameters Measurements}

Data were collected using a structured questionnaire administered face-to-face to participants. The following information was collected: age, gender, self-reported smoking and ethanol drinking habits, physical activity, fruits and vegetables consumption and sleep profile. Physical activity was evaluated from answers to questions on type and frequency of physical exercise (at work and during leisure time) and was categorized into "active" $(\geq 150 \mathrm{~min} /$ week of aerobic exercise such as jogging, swimming, cycling, etc.) or "inactive". Fruits and vegetables intake was considered insufficient if consumption or either fruits or vegetables was $<5$ servings per day [19]. Sleep quality was evaluated using the standard Pittsburgh Sleep Quality Index (PSQI), a widely used measure of sleep quality [20]. This self-rated questionnaire evaluates sleep quality and disturbances over a 1-month period. It has 19 individual items with seven "component" scores. The scores of each item of the index vary between 0 and 3 . The summative score of these seven components ranges between 0 and 21 . Poor sleep quality was defined as a PSQI score $>5$ [20]. Sleep duration was evaluated from responses to relevant questions on actual sleep duration for each day over the past month and classified into "short" ( $\leq 6 \mathrm{~h})$, "normal" $(7 \mathrm{~h} \leq$ sleep duration $\leq 8 \mathrm{~h})$ or "long" $(>8 \mathrm{~h})$ based on previous reports $[21,22]$.

\subsection{Parameters Measurements}

Physical examination included blood pressure (BP), heart rate (HR), waist circumference (WC), weight, and height. Body mass index (BMI) was calculated as weight in kilograms divided by the square of height in meter $\left(\mathrm{kg} / \mathrm{m}^{2}\right)$. Overweight was defined as BMI $\geq 25 \mathrm{~kg} / \mathrm{m}^{2}$, and obesity as BMI $>30 \mathrm{~kg} / \mathrm{m}^{2}$. Abdominal obesity was defined as $\mathrm{WC} \geq$ $102 \mathrm{~cm}$ for men and $\geq 88 \mathrm{~cm}$ for women [23].

BP was measured after 15 minutes in the sitting position and in standardized conditions. Three consecutive BP measurements were taken at 5 minutes intervals using a validated automated sphygmomanometer (HEM-705 CP, Omron Corporation, Tokyo, Japan) with cuff's width adjusted to arm's circumference. A fourth measurement was obtained if the first three readings differed by $\geq 10 \mathrm{mmHg}$. The averages of the nearest three BP and HR readings were considered in this study. Hypertension was defined as systolic BP $\geq 140 \mathrm{mmHg}$ and/or diastolic $\mathrm{BP} \geq 90 \mathrm{mmHg}$, and/or ongoing antihypertensive medication [23].

Capillary blood glucose (CBG) was determined using a glucometer (Accu-Chek Aviva, Roche, Mannheim, Germany). It was not feasible to assert whether participants had observed 8 hours overnight fasting, and as a result the BG values obtained were considered as casual blood glucose (CBG). Diabetes was defined according to American Diabetes Association (ADA) criteria [24], ie CBG $\geq 200$ $\mathrm{mg} / \mathrm{dL}$ and/or being on glucose-lowering medication (s).

\subsection{Statistical Analysis}

Continuous variables are presented as means \pm 1 standard 
deviation (SD), and categorical data as percentages. Prevalence rates are presented with $95 \%$ confidence intervals (CI). The significance of differences between proportions was assessed using Chi squared test (for categorical variables), whereas the significance of differences between normally-distributed continuous variables was assessed using Student's t test. Multivariable logistic regression was used to assess the association between diabetes and sleep quality or duration. Odd ratios were adjusted for age and gender. Statistical significance was set at $\mathrm{p}<0.05$. All analyses were performed using the SPSS 20 software (SSPS Inc, Chicago, Illinois, USA).

\section{Results}

Mean age of the sampled population was $36 \pm 12$ years, and $39.1 \%$ of participants were men. Sociodemographic, anthropometric, bio-clinical parameters, as well as $\mathrm{CV}$ risk factors are presented in Table 1, which also shows the characteristics of urban vs. rural participants. Mean age was higher in rural participants than in those from urban area $(p=0.037)$, whereas the proportion of men was not different between urban and rural participants $(p=0.971)$. Overweight/obesity, abdominal obesity, hypertension, diabetes and related variables were not significantly different between urban and rural areas $(\mathrm{p}>0.05)$. Alcohol consumption and physical activity were more frequent in rural area $(p=0.047$ and $p=0.029$ respectively), where fruits/vegetables consumption was also more frequent than in urban area $(\mathrm{p}=0.0005)$.

Figure 1 and Figure 2 show the distribution of Pittsburgh Sleep Quality Index (PSQI) score and subjective sleep duration (SD) among participants.

Frequency of poor sleep quality (PSQI score $>5$ ) was $50.3 \%$ and was not significantly different between urban and rural participants $(48.2 \%$ vs $52.4 \%$, respectively; $\mathrm{p}=0.395$ ). Participants with short sleep duration (sleep duration $\leq 6 \mathrm{~h}$ ) represented $30.5 \%$ of the sample. Those with sleep duration between $7-8 \mathrm{~h}$ and $>8 \mathrm{~h}$ amounted to $51.7 \%$ and $17.8 \%$, respectively. Frequency of short sleep duration in urban area was $36.1 \%$, a figure significantly higher than in rural area $(24.8 \%) ; \mathrm{p}=0.006$.

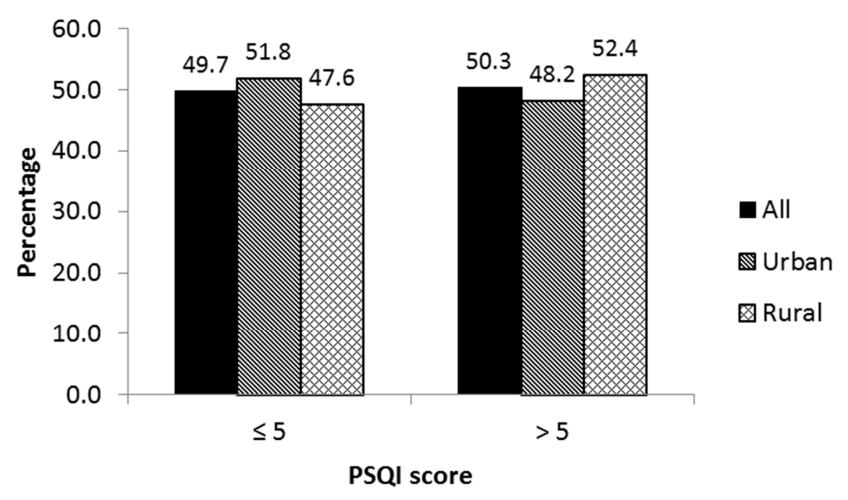

Figure 1. PSQI score in the whole sample, and in urban and rural areas.

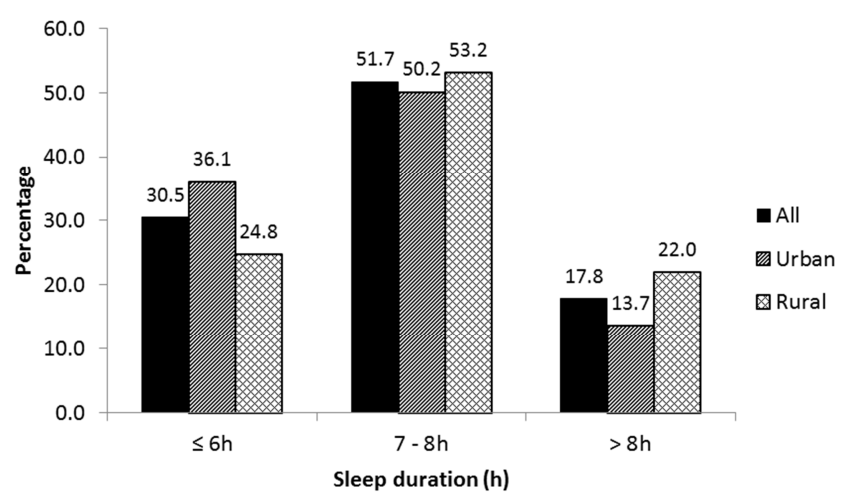

Figure 2. Distribution of sleep duration in the whole study population, and in urban and rural areas.

Table 1. Characteristics of the study participants.

\begin{tabular}{lllll}
\hline & All (N=499) & $\begin{array}{l}\text { Urban } \\
(\mathbf{2 5 0})\end{array}$ & $\begin{array}{l}\text { Rural } \\
(\mathbf{N = 2 4 9 )}\end{array}$ & p \\
\hline Age, years & $36 \pm 12$ & $35 \pm 12$ & $37 \pm 11$ & 0.037 \\
Male gender, \% & $195(39.1)$ & $97(39.0)$ & $98(39.2)$ & 0.971 \\
Weight, kg & $72.8 \pm 15.4$ & $74.2 \pm 15.4$ & $71.5 \pm 15.4$ & 0.051 \\
Height, m & $1.66 \pm 0.08$ & $1.66 \pm 0.08$ & $1.66 \pm 0.08$ & 0.227 \\
BMI, kg/m & $26.4 \pm 5.4$ & $26.8 \pm 5.7$ & $26.0 \pm 5.2$ & 0.111 \\
Overweight/obesity, \% & $268(53.7)$ & $144(57.8)$ & $124(49.6)$ & 0.079 \\
Abdominal obesity, \% & $96(19.2)$ & $50(20.1)$ & $46(18.4)$ & 0.717 \\
Systolic BP, mmHg & $119 \pm 19$ & $119 \pm 19$ & $120 \pm 19$ & 0.326 \\
Diastolic BP, mmHg & $76 \pm 12$ & $75 \pm 13$ & $77 \pm 12$ & 0.195 \\
Hypertension, \% & $104(20.8)$ & $52(20.9)$ & $52(20.8)$ & 0.930 \\
Blood glucose, g/L & $1.07 \pm 0.34$ & $1.06 \pm 0.35$ & $1.08 \pm 0.34$ & 0.405 \\
Diabetes, \% & $41(8.2)$ & $25(10.0)$ & $16(6.4)$ & 0.188 \\
Smoking, \% & $36(7.2)$ & $15(6.0)$ & $21(8.4)$ & 0.394 \\
Alcohol, \% & $116(23.2)$ & $48(19.3)$ & $68(27.2)$ & 0.047 \\
Fruits/ vegetables, \% & $120(24.0)$ & $77(30.9)$ & $43(17.2)$ & 0.0005 \\
Physical activity, \% & $323(64.7)$ & $149(59.8)$ & $174(69.6)$ & 0.029 \\
Sleep duration, hour & $6.3 \pm 1.4$ & $6.1 \pm 1.4$ & $6.6 \pm 1.4$ & 0.0002 \\
PSQI total score & $6.1 \pm 2.9$ & $6.1 \pm 3.1$ & $6.1 \pm 2.6$ & 0.989 \\
\hline
\end{tabular}

BMI: body mass index; BP: blood pressure; PSQI: Pittsburgh sleep quality index. Quantitative variables are presented as mean \pm 1 standard deviation; qualitative variables are shown as number of subjects, with values in brackets representing percentages. $P$ values represent the statistical significance of differences between urban and rural participants.

In the whole sample, frequency of diabetes was significantly higher in participants with $\mathrm{SD} \leq 6 \mathrm{~h}$ compared to those with $\mathrm{SD}>6 \mathrm{~h}(\mathrm{p}<0.001)$. Although the frequency of diabetes was higher in participants with poor sleep quality (PSQI $>5$ ) compared to those with PSQI $\leq 5$ (10\% vs $6.5 \%$ respectively), the difference was not significant $(p>0.05)$ When combining sleep quality with $\mathrm{SD}$, participants with normal sleep quality associated with short SD (PSQI $\leq 5+\mathrm{SD}$ $\leq 6 \mathrm{~h}$ ) and those with poor sleep quality associated with short $\mathrm{SD}$ (PSQI $>5+\mathrm{SD} \leq 6 \mathrm{~h}$ ) had a higher risk of prevalent diabetes compared to those with both normal sleep quality and normal SD. On the basis of geographical origin, results were about the same in urban area than in the overall population, but no significant association was found in rural area. After adjustment for age and sex, there was a significant association between short SD and diabetes in the whole sample (OR 2.53 95\%CI1.32-4.85; $<<0.01$ ). Associations of short SD with poor and normal sleep quality were also significantly associated with prevalent diabetes (OR: 4.12 
95\%CI 1.18-14.4; $\mathrm{p}<0.05$ and OR $2.6795 \%$ CI 1.22- 5.81; $\mathrm{p}<0.05$, respectively).

Table 2. Relation of diabetes with sleep quality and sleep duration in the whole sample.

\begin{tabular}{|c|c|c|c|c|}
\hline & & n (\%) & OR $(95 \%$ CI $)$ & AOR (95\% CI) \\
\hline & All & & & \\
\hline \multirow[t]{2}{*}{ SD } & $\mathrm{SD}>6 \mathrm{~h}$ & $20(5.8)$ & Ref & Ref \\
\hline & $\mathrm{SD} \leq 6 \mathrm{~h}$ & $21(13.8)$ & $2.62(1.38-5.00)^{* * *}$ & $2.53(1.32-4.85)^{* *}$ \\
\hline \multirow[t]{2}{*}{ PSQI Score } & $\mathrm{PSQI} \leq 5$ & $16(6.5)$ & Ref & Ref \\
\hline & $\mathrm{PSQI}>5$ & $25(10.0)$ & $1.60(0.83-3.08)$ & $1.68(0.87-3.25)$ \\
\hline \multirow[t]{4}{*}{$\mathrm{PSQI}+\mathrm{SD}$} & $\mathrm{PSQI} \leq 5+\mathrm{SD}>6 \mathrm{~h}$ & $12(5.3)$ & Ref & Ref \\
\hline & $\mathrm{PSQI}>5+\mathrm{SD}>6 \mathrm{~h}$ & $8(6.7)$ & $1.28(0.51-3.22)$ & $1.41(0.55-3.56)$ \\
\hline & $\mathrm{PSQI} \leq 5+\mathrm{SD} \leq 6 \mathrm{~h}$ & $4(19.0)$ & $4.22(1.23-14.49)^{*}$ & $4.12(1.18-14.4)^{*}$ \\
\hline & $\begin{array}{l}\mathrm{PSQI}>5+\mathrm{SD} \leq 6 \mathrm{~h} \\
\text { Urban }\end{array}$ & $17(13.0)$ & $2.67(1.23-5.79)^{*}$ & $2.67(1.22-5.81)^{*}$ \\
\hline \multirow[t]{2}{*}{ SD } & $\mathrm{SD}>6 \mathrm{~h}$ & $10(6.3)$ & Ref & Ref \\
\hline & $\mathrm{SD} \leq 6 \mathrm{~h}$ & $15(16.7)$ & $2.98(1.28-6.95)^{*}$ & $3.02(1.29-7.10)^{*}$ \\
\hline \multirow[t]{2}{*}{ PSQI Score } & $\mathrm{PSQI} \leq 5$ & $10(7.8)$ & Ref & Ref \\
\hline & $\mathrm{PSQI}>5$ & $15(12.5)$ & $1.70(0.73-3.95)$ & $1.77(0.76-4.15)$ \\
\hline \multirow[t]{5}{*}{$\mathrm{PSQI}+\mathrm{SD}$} & $\mathrm{PSQI} \leq 5+\mathrm{SD}>6 \mathrm{~h}$ & $7(6.2)$ & Ref & Ref \\
\hline & $\mathrm{PSQI}>5+\mathrm{SD}>6 \mathrm{~h}$ & $3(6.5)$ & $1.06(0.26-4.28)$ & $1.13(0.28-4.60)$ \\
\hline & $\mathrm{PSQI} \leq 5+\mathrm{SD} \leq 6 \mathrm{~h}$ & $3(18.8)$ & $3.49(0.80-15.2)$ & $3.61(0.81-15.9)$ \\
\hline & $\mathrm{PSQI}>5+\mathrm{SD} \leq 6 \mathrm{~h}$ & $12(16.2)$ & $2.93(1.10-7.84)^{*}$ & $3.03(1.12-8.16)^{*}$ \\
\hline & Rural & & & \\
\hline \multirow[t]{2}{*}{ SD } & $\mathrm{SD}>6 \mathrm{~h}$ & $10(5.3)$ & Ref & Ref \\
\hline & $\mathrm{SD} \leq 6 \mathrm{~h}$ & $6(9.7)$ & $1.91(0.66-5.48)$ & $1.72(0.59-5.04)$ \\
\hline \multirow[t]{2}{*}{ PSQI Score } & $\mathrm{PSQI} \leq 5$ & $6(5.0)$ & ref & Ref \\
\hline & PSQI > 5 & $10(7.6)$ & $1.56(0.55-4.42)$ & $1.61(0.56-4.63)$ \\
\hline \multirow[t]{4}{*}{ PSQI + SD } & $\mathrm{PSQI} \leq 5+\mathrm{SD}>6 \mathrm{~h}$ & $5(4.4)$ & Ref & Ref \\
\hline & $\mathrm{PSQI}>5+\mathrm{SD}>6 \mathrm{~h}$ & $5(6.8)$ & $1.58(0.44-5.66)$ & $1.75(0.48-6.35)$ \\
\hline & $\mathrm{PSQI} \leq 5+\mathrm{SD} \leq 6 \mathrm{~h}$ & $1(20.0)$ & $5.45(0.51-58.2)$ & $4.66(0.43-50.8)$ \\
\hline & $\mathrm{PSQI}>5+\mathrm{SD} \leq 6 \mathrm{~h}$ & $5(8.8)$ & $2.10(0.58-7.56)$ & $1.98(0.54-7.25)$ \\
\hline
\end{tabular}

PSQI: Pittsburgh sleep quality index; SD: sleep duration; OR: odd ratio; AOR: adjusted odd ratio (adjusted for age and sex); CI: confidence interval; *p $<0.05$; $* * \mathrm{p}<0.01 ; * * * \mathrm{p}<0.001$

\section{Discussion}

In this cross-sectional comparative study, we found some important results underlying the association between sleep quality and prevalent type 2 diabetes mellitus in urban and rural communities in two Cameroonian populations. Mean sleep duration in the whole sample was $6.3 \mathrm{~h}$, and was significantly longer in rural vs. urban participants, whereas the frequency of short sleep duration was significantly higher in urban participants. Contrary to what might have been expected, the frequency of poor sleep quality was similar in urban and rural areas [25]. As expected from the existing literature, short sleep duration was significantly associated with risk of prevalent diabetes in the whole sample. Although poor sleep quality was not significantly associated with diabetes, the combination of short sleep duration with poor sleep quality was significantly associated with the presence of diabetes.

Short sleep duration $(\leq 6 \mathrm{~h})$ as well as poor sleep quality (PSQI $>5$ ) were previously reported by numerous authors as predictive factors for various cardio metabolic comorbidities such as hypertension [26], metabolic syndrome [27] and overweight/obesity [28].

The frequency of poor sleep quality found in the present study was relatively high compared to those reported from other sub-Saharan African countries. A large study conducted in $>40000$ adults from 8 African and Asian countries showed that prevalence of poor sleep quality ranged from 8.3 to $12.7 \%$ in Kenya, Ghana and Tanzania. In South Africa, the prevalence was $31.3 \%$ in women and $27.3 \%$ in men [29]. Conversely, the present data are similar to those of Tai et al. who found a prevalence of $46.6 \%$ in a Taiwanese population [30].

There is a bundle of literature on the association between type 2 diabetes mellitus and sleep disorders using different sleep quality evaluation tools. Many authors have demonstrateda high prevalence of sleep disorders in participants with diabetes mellitus compared to those without diabetes. Using the Pittsburgh Sleep Quality Index (PSQI), Fiorentini et al. reported a higher prevalence of diabetes mellitus in participants with poor sleep quality $(19.4 \%)$ compared to those with good sleep quality (8.8\%) [31]. More recently, Zubair et al. found that PSQI global score as well as PSQI score $>5$ were higher or more frequent, respectively, in participants with compared to those without diabetes [32]. We found in the present study a 2.62-fold increased risk of prevalent diabetes in subjects with short sleep duration $(\mathrm{SD} \leq$ $6 \mathrm{~h})$ compared to those with normal sleep duration ( $\mathrm{SD} \geq 6 \mathrm{~h}$ ), consistent with the results of Anothaisinta wee et al. who showed that short sleep duration was predictive of diabetes with a relative risk of $1.18(95 \%$ CI $1.10-1.26)$ [33]. Short sleep duration was also shown to be a strong predictive factor for impaired fasting glucose and/or impaired glucose tolerance. Engeda et al., found that individuals who reported short sleep duration had 2.06 higher odds of prediabetes 
(95\%CI 1.00-4.22) compared to individuals who reported $7 \mathrm{~h}$ of sleep [34].

The mechanisms underlying the association between abnormal sleep quality and duration and abnormalities in glucose homeostasis are not well established. These may include higher evening cortisol levels, increased sympathetic nervous system activity and catecholamine production, as well as higher levels of inflamatory factors, which may promote insulin resistance, such as interleukin (IL)-1 $\beta$, IL-6, IL-17, and C-reactive protein [35]. In addition, several shortterm studies showed that sleep fragmentation or restriction promotes to insulin resistance $[36,37]$ which appears to play a key role in the pathophysiology of diabetes in predisposed subjects who are unable over a lifetime to sustain compensatory hyperinsulinaemia. Although the frequency of diabetes in this study was higher in participants with poor sleep quality (PSQI $>5$ ) compared to those with normal sleep quality ( $10 \%$ vs $6.5 \%$ respectively), the association of sleep quality and diabetes was not statistically significant. Hung et al. previously reported that hyperglycaemia is an independent determinant of the global PSQI score [27]. In addition, other studies showed that poor sleep quality were associated with poor glycaemic control and development of type 2 diabetes $[14,18]$.

When combining sleep duration with sleep quality, we found that participants with short sleep duration and normal sleep quality as well as those with short sleep duration and poor sleep quality had a high risk of prevalent diabetes compared to those with normal sleep duration and quality (OR 4.22, 95\%CI 1.23- 14.49 and OR 2.67, 1.23- 5.79 respectively). The higher odds of having diabetes when combining reduced sleep duration and quality rather than looking at them separately emphasizes the necessity of considering those two parameters together in the management of cardiovascular disorders. Our results are corroborated by previous studies aiming to explore the interaction between sleep duration/quality and impaired fasting glucose [13], hypertension [26] and CV events [38], which also suggest some additive effects. Yet, the present study is the first demonstration of the interaction between sleep quality, sleep duration and type 2 diabetes in a mixed Cameroonian population.

This study has several methodological limitations. The patients were recruited in two local urban and rural communities and cannot de facto be representative of other Cameroonian populations. As this was a cross-sectional survey, one cannot establish the directionality and causality of the observed associations between sleep disorders and diabetes. In addition, it was not feasible to formally establish the presence/absence of primary sleep disorders such as Obstructive Sleep Apnoea Syndrome and Restless Legs Syndrome, both of which could have modulated and impacted on the observed results.

\section{Conclusion}

This cross-sectional study shows a high prevalence of short sleep duration and poor sleep quality in an urban and rural community of Cameroon. Whereas diabetes was not significantly associated with poor sleep quality, it was more frequent in participants with poor sleep quality compared to those with normal sleep quality in this mixed Cameroonian population. Combining sleep quality with sleep duration, participants with normal sleep quality associated with short sleep duration and those with poor sleep quality associated with short sleep duration had a higher risk of prevalent diabetes compared to those who had both normal sleep duration and quality. These findings highlight the value of considering different aspects of sleep disorders, and in particular their factorial association, in assessing their contribution to the various features of cardio metabolic risk.

\section{References}

[1] Bonnet MH, Arand DL. We are chronically sleep deprived. Sleep1995; 18: 908-11.

[2] Knutson KL, Van Cauter E, Rathouz PJ, DeLeire T, Lauderdale DS. Trends in the prevalence of short sleepers in the USA: 1975-2006. Sleep 2010; 33 (1): 37-45.

[3] Leger D, Roscoat E, Bayon V, Guignard R, Paquereau J, Beck F. Short sleep in young adults: Insomnia or sleep debt? Prevalence and clinical description of short sleep in a representative sample of 1004 young adults from France. Sleep Med. 2011; 12 (5): 454-462.

[4] Brown WD. Insomnia: Prevalence and daytime consequences. In: Lee-Chiong T, editor. Sleep: A Comprehensive Handbook. Hoboken, New Jersey: John Wiley and Sons; 2006. pp. 93-98.

[5] Trenell MI, Marshall NS, Rogers NL. Sleep and metabolic control: waking to a problem? Clin. Exp. Pharmacol. Physiol. 2007; 34: 1-9.

[6] National Sleep Foundation (NSF). Sleep in America Poll 2003. Washington, DC: National Sleep Foundation; 2003

[7] Mitler M, Carskadon MA, Czeisler CA, Dement WC, Dinges DF, GraeberRC. Catastrophes, sleep, and public policy: Consensus report. Sleep 1988; 11: 100-9.

[8] Gilling JC. Are sleep disturbances risk factors for anxiety, depressive and addictive disorders? Acta Psychiatr Scand Suppl 1998; 393: 39-43.

[9] Colten HR, Altevogt BM, editors. Sleep disorders and sleep deprivation: An unmet public health problem. Washington DC: National Academies Press; 2006. p. 67-209.

[10] Marjollet A., Weiszenstein M., Henri M., Thomas A., Ribuot D., et al, The impact of sleep disorders on glucose metabolism: endocrine and molecular mechanisms, Diabetol. Metab. Syndr. 2015; 25: 7-25.

[11] Logue EE, Scott ED, Palmieri PA, Dudley P. Sleep duration, quality, or stability and obesity in an urban family medicine center. J. Clin. Sleep Med. 2014; 10: 177-182.

[12] Okubo N, Matsuzaka M, Takahashi I, Sawada K, Sato S, Akimoto N, Umeda T, Nakaji S. Relationship between selfreported sleep quality and metabolic syndrome in general population. BMC Public Health 2014; 14: 562. 
[13] Lou P, Chen P, Zhang L, Zhang P, Chang G, Zhang N, Li T, Qiao C. Interaction of sleep quality and sleep duration on impaired fasting glucose: A population-based cross-sectional survey in China. BMJ Open 2014, 4.

[14] Cappuccio FP, D'Elia L, Strazzullo P, Miller MA. Quantity and quality of sleep and incidence of type 2 diabetes: a systematic review and meta-analysis. Diabetes Care. 2010; 33: 414-420. DOI: $10.2337 / \mathrm{dc} 09-1124$.

[15] Holliday EG, Magee CA, Kritharides L, Banks E, Attia J. Short sleep duration is associated with risk of future diabetes but not cardiovascular disease: a prospective study and metaanalysis. PLoS One. 2013; 8: e82305. doi: 10.1371/journal.pone.0082305.

[16] Broussard JL, Ehrmann DA, Van Cauter E, Tasali E, Brady MJ. Impaired insulin signaling in human adipocytes after experimental sleep restriction: a randomized, crossover study. Ann Intern Med. 2012; 157: 549-557. DOI: 10.7326/00034819-157-8-201210160-00005.

[17] Buxton OM, Pavlova M, Reid EW, Wang W, Simonson DC, Adler GK. Sleep restriction for 1 week reduces insulin sensitivity in healthy men. Diabetes. 2010; 59: 2126-2133. DOI: $10.2337 / \mathrm{db} 09-0699$.

[18] Knutson KL, Van Cauter E, Zee P, Liu K, Lauderdale DS. Cross-sectional associations between measures of sleep and markers of glucose metabolism among subjects with and without diabetes: the Coronary Artery Risk Development in Young Adults (CARDIA) Sleep Study. Diabetes Care. 2011; 34 (5): 1171-6.

[19] World Health Organization (WHO). Global Status Report on noncommunicable diseases 2010. Geneva: World Health Organization, 2011.

[20] Buysse DJ, Reynolds CF 3rd, Monk TH, Berman SR, Kupfer DJ. The Pittsburgh Sleep Quality Index: a new instrument for psychiatric practice and research. Psychiatry Res 1989; 28: 193-213.

[21] Martica, H. H.; Muldoon, M. F.; Jennings, J. R.; Buysse, D. J.; Flory, J. D.; Manuck, S. B. Self-reported sleep duration is associated with the metabolic syndrome in midlife adults. Sleep 2008; 31: 635-643.

[22] James EG, Heymsfield SB, Boden-Albala B, Buijs RM, Kreier F, Pickering TG, Rundle AG, Zammit GK, Malaspina D. Short sleep duration as a risk factor for hypertension analyses of the first national health and nutrition examination survey. Hypertension 2006; 47: 833-839.

[23] Mancia G, Fagard R, Narkiewicz K, et al. 2013 ESH/ESC hypertension guidelines for the Management of Arterial Hypertension: the Task Force for the Management of Arterial Hypertension of the European Society of Hypertension (ESH) and of the European Society of cardiology (ESC). Eur Heart J. 2013; 34: 2159-2219.

[24] American Diabetes Association (ADA). Standards of medical care in diabetes-2010. Diabetes Care 2010; 33: 11-61.

[25] Hsu YW, Ho CH, Wang JJ, Hsieh KY, Weng SF, Wu MP.
Longitudinal trends of the healthcare-seeking prevalence and incidence of insomnia in Taiwan: an 8-year nationally representative study. Sleep Med. 2013; 14: 843-9.

[26] Lu K, Chen J, Wang L, Wang C, Ding R, Wu S Hu D. Association of Sleep Duration, Sleep Quality and Shift-Work Schedule in Relation to Hypertension Prevalence in Chinese Adult Males: A Cross-Sectional Survey. Int. J. Environ. Res. Public Health 2017; 14: 210; doi: 10.3390/ijerph14020210.

[27] Hung H-C, Yang Y-C, Ou H-Y, Wu J-S, Lu F-H, Chang CJ. The Association between Self-Reported Sleep Quality and Metabolic Syndrome. PLoS ONE 2013; 8 (1): e54304. doi: 10.1371/journal.pone.0054304.

[28] Ford ES, Li C, Wheaton AG, Chapman DP, Perry GS, Crof JB. Sleep duration and body mass index and waist circumference among US adults. Obesity 2014; 22 (2): 598 607. doi: 10.1002/oby.20558.

[29] Stranges S, Tigbe W, Gomez-Olivé FX, Thorogood M, Kandala NB. Sleep problems: an emerging global epidemic? Finding from the INDEPTH WHO-SAGE study among more than 40,000 older adults from 8 countries across Africa and Asia. Sleep Med Rev. 2012; 35 (8): 1173-81.

[30] Tai SY, Wang WF, Yang YH. Current status of sleep quality in Taiwan: a nationwide walk-in survey. Ann Gen Psychiatry 2015; 14: 36 DOI 10.1186/s12991-015-0078-7.

[31] Fiorentini A, Valente R, Perciaccante A, Tubani L. Sleep's quality disorders in patients with hypertension and type 2 diabetes mellitus. Int J Cardiol. 2007; 114 (2): E50-2.

[32] Zubair U, Majid F, Siddiqui A A, Zubair Z. Sleep Abnormalities Among Patients With and Without Diabetes Using Pittsburg Sleep Quality Index and Epworth Sleepiness Scale. Cureus 2018; 10 (2): e2151. DOI 10.7759/cureus.2151.

[33] Anothaisintawee T, Reutrakul S, Van Cauter E, Thakkinstian A. Sleep disturbances compared to traditional risk factors for diabetes development: systematic review and meta-analysis. Sleep Med Rev. 2015; 30: 11-24.

[34] Engeda J, Mezuk B, Ratliff S, Ning Y. Association between duration and quality of sleep and the risk of pre-diabetes: evidence from NHANES. Diabet Med. 2013; 30 (6): 676-80.

[35] Knutson KL. Sociodemographic and cultural determinants of sleep deficiency: implications for cardiometabolic disease risk. Soc Sci Med 2013; 79: 7-15. doi: 10.1016/j.socscimed.2012.05.002.

[36] Tasali E, Leproult R, Ehrmann DA, Van CauterE. Slow-wave sleep and the risk of type 2 diabetes in humans. Proc Natl AcadSci USA 2008; 105: 1044-1049.

[37] Stamatakis KA, Punjabi NM. Effects of sleep fragmentation on glucose metabolism in normal subjects. Chest 2010; 137: 95-101.

[38] Hoevenaar-Blom MP, Spijkerman AM, Kromhout D, van den Berg JF, Verschuren WM. Sleep duration and sleep quality in relation to 12-year cardiovascular disease incidence: The MORGEN study. Sleep 2011; 34: 1487-1492. 